\title{
miR-205 suppresses cell proliferation, invasion, and metastasis via regulation of the PTEN/AKT pathway in renal cell carcinoma
}

\author{
HUIQIANG WANG $^{1 *},{\text { BIN } \text { CHEN }^{1 *}, \text { BO DUAN }}^{1}$, JIAXIN ZHENG $^{1}$ and XINYI WU ${ }^{2}$ \\ Departments of ${ }^{1}$ Urology and ${ }^{2}$ Breast Surgery, First Affliated Hospital of Xiamen University, \\ Xiamen, Fujian 361003, P.R. China
}

Received July 14, 2015; Accepted July 20, 2016

DOI: $10.3892 / \mathrm{mmr} .2016 .5589$

\begin{abstract}
The present study aimed to determine the importance of microRNA-205 (miR-205) in the proliferation, apoptosis, invasion and metastasis of renal cell carcinoma (RCC) cells and the underlying molecular mechanisms. Reverse transcription-polymerase chain reaction was used to quantify the expression levels of miR-205 in RCC tissue, normal tissue adjacent to carcinoma, RCC cells and normal renal cells. It was determined that the expression levels of miR-205 in RCC tissue and cells were reduced compared with those in normal tissue and renal cells. miR-205 mimics and the negative control were prepared and transfected into RCC cells. Cell viability and apoptosis were investigated using methyl thiazolyl tetrazolium assay and Annexin V-fluorescein isothiocyanate/propidium iodide staining, respectively. Cell migration and invasion were evaluated with Transwell assays. The protein expression levels of E2F transcription factor 1 (E2F1), B-cell lymphoma-2 (Bcl-2), E-cadherin, vimentin, phosphatase and tensin homolog (PTEN) and phosphorylated AKT serine/threonine kinase 1 (p-AKT) were determined with western blot analysis. It was revealed that miR-205 promoted the apoptosis of RCC cells and suppressed their proliferation, metastasis and invasion compared with the negative control. The expression levels of E2F1, Bcl-2, vimentin and p-AKT were downregulated compared with the negative control. The expression levels of E-cadherin and PTEN were upregulated in the cells transfected with miR-205 mimics compared with the negative control group. Therefore, it was concluded that miR-205 suppressed cell proliferation, invasion, and metastasis in RCC cells via regulation of the PTEN/AKT
\end{abstract}

Correspondence to: Professor Xinyi Wu, Department of Breast Surgery, First Affliated Hospital of Xiamen University, 55 Zhenhai Road, Xiamen, Fujian 361003, P.R. China

E-mail: wuxinyixu@163.com

*Contributed equally

Key words: miR-205, renal cell carcinoma, cell proliferation, tumor metastasis, tumor invasion, PTEN/AKT signaling pathway signaling pathway. The present study may contribute to future miRNA-based RCC therapy.

\section{Introduction}

Renal cell carcinoma (RCC) is a type of malignant tumor, which originates from renal tubular epithelial cells. It poses a serious threat to human health, as its morbidity rate is high among tumors of the urinary system $(1,2)$. In recent years, the morbidity and mortality rates of patients with RCC have increased in China (3). Radical nephrectomy is currently the most effective treatment for RCC. The 5-year survival rate of patients following surgery is currently $<10 \%$, despite efforts to improve it (2). Therefore, the development of novel effective treatment would be of great significance for patients with RCC. Notably, multiple microRNAs (miRNAs) have been identified to be closely associated with the genesis and development of RCC, including effects on proliferation, apoptosis, invasion and metastasis, which may provide an alternative strategy for the early diagnosis and prognosis of RCC.

Endogenous non-coding miRNAs have highly conserved sequences; therefore, they are able to inhibit the expression of a target gene by binding to the 3'-untranslated region of the mRNA and, subsequently, inhibiting its translation. Over 700 human miRNAs have been identified to participate in $\sim 1 / 3$ of human gene expression. Additionally, the abnormal expression of certain miRNAs in RCC had been confirmed by previous studies (4-6). Gottardo et al (4) identified 10 miRNAs, including miR-223, miR-26b, miR-221, miR-103-1, miR-185, miR-23b, miR-203, miR-17-5p, miR-23a and miR-205, that were highly expressed in RCC when compared with normal nephridial tissue (4). Chow et al (5) also determined that the expression levels of 21 miRNAs were upregulated, and 12 miRNAs were downregulated, in RCC (5). Similarly, the abnormal expression of 86 miRNAs in RCC was investigated by Yi et al (6) using gene chip technology, where 48 miRNAs were highly expressed and 38 exhibited low expression levels (6). Therefore, specific regulation of the target gene by diverse miRNAs may contribute to the improvement of RCC treatment.

miRNA-205 (miR-205) sequence was first calculated from homogenous sequences of mice and Takifugu rubripes using miRNA software (7). In humans, miR-205 is located on the second intron of the long arm of chromosome 1 and 
is not located near to other miRNAs. miR-205 has been determined to be abnormally expressed in various types of tumor. miR-205 is capable of acting as an oncogene and tumor suppressor. As a tumor suppressor, it may regulate the malignant biological behaviors of various types of tumor, including prostatic carcinoma, squamous cell carcinoma of the head and neck and breast carcinoma, via targeting the expression of various genes $(8,9)$. Previous studies have reported low expression levels of miR-205 in RCC $(10,11)$, with miR-205 levels important for the regulation of RCC (11). However, the detailed molecular mechanisms involved remain to be elucidated. Therefore, the present study aimed to validate the low expression levels of miR-205 in the tissue and cells of RCC, investigate the effect of miR-205 on the malignant biological behaviors of RCC, including cell proliferation, invasion and metastasis, and determine the underlying molecular mechanisms.

\section{Materials and methods}

Specimen collection. Ethical approval and written informed consent was obtained from the Drug Clinical Trials Ethics Committee, First Affliated Hospital of Xiamen University (Fujian, China), and 20 RCC and 20 normal tissues adjacent to carcinoma were collected from patients that underwent laparoscopic radical nephrectomy in the Department of Urology, First Afflicted Hospital of Xiamen University (Xiamen, China), between May 2014 and May 2015. Patients did not receive chemotherapy or radiotherapy prior to the operation and pathological diagnosis was performed.

Materials and cell culture. The Annexin V-fluorescein isothiocyanate (FITC)/propidium iodide (PI) apoptosis detection kit was obtained from Keygen Biotech Co., Ltd. (Nanjing, China). Bicinchoninic acid assay (BCA) kit was from Beyotime Institute of Biotechnology (Nanjing, China). Lipofectamine 2000, TRIzol reagent and one-step reverse transcription-polymerase chain reaction (RT-PCR) kit were purchased from Invitrogen; Thermo Fisher Scientific, Inc. (Waltham, MA, USA). Methyl thiazolyl tetrazolium (MTT) was purchased from Gibco (Thermo Fisher Scientific, Inc.). Rabbit anti-E2F transcription factor 1 (E2F1; cat. no. 8120-1), anti-B-cell lymphoma-2 (Bcl-2 ; cat. no. 1017-1), anti-E-cadherin (cat. no. 5409-1), anti-vimentin (cat. no. 2707-1) and GAPDH (cat. no. 5632-1) monoclonal antibodies were obtained from Epitomics (Burlingame, CA, USA). Rabbit anti-phosphatase and tensin homolog (PTEN; cat. no. 9552), anti-AKT serine/threonine kinase 1 (AKT; cat. no. 9272) and anti-phosphorylated AKT (p-AKT; cat. no. 4060) antibodies were purchased from Cell Signaling Technology, Inc. (Danvers, MA, USA). Horseradish peroxidase-conjugated goat anti-rabbit antibody (cat. no. A0208) was obtained from Beyotime Institute of Biotechnology, Inc. Transwell inserts were obtained from Corning Incorporated (Corning, NY, USA).

Human RCC cell lines, ACHN, 769-p, and Caki-1, were purchased from the Type Culture Collection of the Chinese Academy of Sciences (Shanghai, China; cat. nos. TCHu199, TCHu215 and TCHu135, respectively). Normal human renal tubular epithelial cell line HK-2 was purchased from American Type Culture Collection (Manassas, VA, USA). Cells were cultured in Dulbecco's modified Eagle's medium (DMEM; GE Healthcare Life Sciences, Logan, UT, USA) supplemented with $10 \%$ (v/v) fetal bovine serum (FBS; GE Healthcare Life Sciences).

Preparation of miRNA. miR-205 mimics and the negative control were synthesized by GenePharma Co., Ltd., (Shanghai, China). The sequences were as follows: miR-205 mimics, forward 5'-UCCUUCAUUCCACCGGAGUCUG-3', reverse 5'-GACUCCGGUGGAAUGAAGGAUU-3'; and negative control, forward 5'-UUCUCCGAACGUGUCACGUTT-3', reverse 5'-ACGUGACACGUUCGGAGAATT-3'. The mix ratio of the miRNA and Lipofectamine 2000 reagent for gene transfection was defined according to the manufacturer's protocol.

miR-205 expression level in RCC tissues and cells. Total RNA of RCC tissues and cells was extracted using TRIzol reagent in an RNase-free environment following to the manufacturer's protocol. The reverse transcription from RNA to cDNA and PCR amplification were performed using the one-step RT-PCR kit. The primers used are presented in Table I and were added to $25 \mu \mathrm{l}$ PCR reaction system. The thermocycling parameters were defined as follows: Denaturation for $45 \mathrm{sec}$ at $94^{\circ} \mathrm{C}$, annealing for $45 \mathrm{sec}$ at $59^{\circ} \mathrm{C}$, elongation for $60 \mathrm{sec}$ at $72^{\circ} \mathrm{C}$ for 35 cycles. The amplification products $(5 \mu \mathrm{l})$ were loaded on a $2 \%(\mathrm{w} / \mathrm{v})$ agarose gel for separation by gel electrophoresis. A ChemiDoc XRS gel imaging system (Bio-Rad Laboratories, Inc., Hercules, CA, USA) using Goldview dye for visualization and analysis of electrophoresis strips. Image Pro Plus version 6.0 (Media Cybernetics, Inc., Rockville, MD, USA) was used for quantification.

Cell viability. ACHN cells were seeded at a density of $2.0 \times 10^{4}$ cells/well in 96-well plates. When cell confluence reached 50\%, miR-205 mimics and the negative control were transfected into cells using Lipofectamine 2000. After $48 \mathrm{~h}$, $20 \mu \mathrm{MTT}(5 \mathrm{mg} / \mathrm{ml})$ was added into each well and cells were cultured for a further $4 \mathrm{~h}$. Culture media was discarded and $150 \mu 1$ dimethyl sulfoxide was added into each well. When the crystal was fully dissolved, absorbance was determined at 560 and $630 \mathrm{~nm}$ (reference wavelengths) using a microplate reader (Infinite F200/M200; Tecan, Männedorf, Switzerland). The relative cell viability was calculated. Cells without any treatment served as control.

Cell apoptosis. ACHN cells were seeded at a density of $1.0 \times 10^{4}$ cells/well in 6 -well plates. When cell confluence reached $50 \%$, miR-205 mimics and the negative control were transfected into cells using Lipofectamine 2000. The Annexin V-FITC/PI apoptosis detection kit was used to determine the cell apoptosis according to the manufacturer's protocol. Briefly, following $24 \mathrm{~h}$ of incubation, the cells were digested with $0.25 \%$ (w/v) trypsin, washed with phosphate-buffered saline and collected with a centrifugation at $800 \mathrm{x} g$ for $5 \mathrm{~min}$. Cells were then resuspended in $500 \mu \mathrm{l}$ binding buffer. Cell suspension was mixed with $5 \mu$ l Annexin V-FITC and subsequently with $5 \mu \mathrm{l}$ PI. After $15 \mathrm{~min}$ incubation at room temperature in the dark, cell apoptosis was determined using a flow cytometer and CellQuest Pro version 6.0 software (FACScan; BD Biosciences, Franklin Lakes, NJ, USA). 
Table I. Primers for reverse transcription-polymerase chain reaction.

\begin{tabular}{llc}
\hline Gene & \multicolumn{1}{c}{ Primer (5'-3') } & bp \\
\hline miR-205 & F ACACTCCAGCTGGGTCCTT & 72 \\
& R CTCAACTGGTGTCGTGGA & \\
GADPH & F AGCCACATCGCTCAGACA & 314 \\
& R TGGACTCCACGACGTACT & \\
\hline
\end{tabular}

F, forward; R, reverse; bp, base pairs. miR, microRNA.

Cell migration. ACHN cells were seeded at a density of $1.0 \times 10^{4}$ cells/well in 6 -well plates. When cell confluence reached 50\%, miR-205 mimics and the negative control were transfected into cells with Lipofectamine 2000. Next, cells were digested and transferred into the upper chamber of Transwell insert without Matrigel. DMEM supplemented with 5\% (v/v) FBS was added into the lower chamber of the Transwell insert. Cells were incubated for $24 \mathrm{~h}$. The Transwell insert was removed, washed, fixed in paraformaldehyde, and stained with crystal violet. The number of cells in the lower chamber of 5 visual fields was counted with a light microscope (Eclipse TS100; Nikon Corporation, Tokyo, Japan). The average number of cells in every visual field represented the migration capacity of cells.

Cell invasion. ACHN cells $\left(1.0 \times 10^{4}\right.$ cells/well) were transfected with miR-205 mimics and the negative control. Matrigel was uniformly distributed onto the membrane of the Transwell insert $(5 \mu \mathrm{m})$ prior to addition of the cells. Then Transwell assay was performed in a similar manner to the aforementioned cell migration assay. The average number of cells in every visual field in the lower chamber represented the invasion capacity of cells.

Western blotting. ACHN cells $\left(1.0 \times 10^{4}\right.$ cells/well) were transfected with miR-205 mimics and the negative control. After $48 \mathrm{~h}$ the cells were collected and lysed with radioimmunoprecipitation assay lysis buffer. A $30 \mathrm{sec}$ agitation using a vortex was performed every $10 \mathrm{~min}$. After $40 \mathrm{~min}$, the mixture was centrifuged for $10 \mathrm{~min}$ at $7,000 \mathrm{x} \mathrm{g}$ and $4^{\circ} \mathrm{C}$. The supernatant was carefully collected to obtain the total protein. BCA kit was used to determine the protein content. Protein (30 ng) was loaded onto a sodium dodecyl sulphate-polyacrylamide gel in order to perform electrophoresis. Proteins were transferred to a polyvinylidene difluoride membrane using the wet transfer method. The membrane was blocked with $5 \%(\mathrm{w} / \mathrm{v})$ non-fat dry milk at room temperature for $1 \mathrm{~h}$. The membrane was immersed in the primary antibodies (dilution, 1:100) buffer overnight at $4^{\circ} \mathrm{C}$. Next, it was rinsed and immersed in a secondary antibody buffer (dilution, 1:200) for $2 \mathrm{~h}$ at room temperature. The membrane was rinsed again and enhanced chemiluminescent solution (Beyotime Institute of Biotechnology, Inc.) was dropped onto the membrane followed by exposure on a gel imaging system (ChemiDoc XRS; Bio-Rad Laboratories, Inc.). QuantityOne version 4.6.2 software (Bio-Rad Laboratories, Inc.) was used to determine the gray

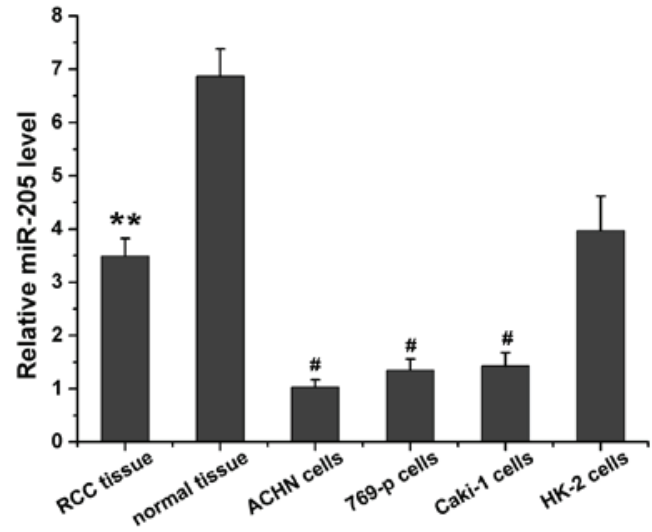

Figure 1. miR-205 expression levels in RCC tissue, normal tissue adjacent to carcinoma, RCC cells (ACHN, 769-p, and Caki-1), and normal renal cells (HK-2) evaluated by semi-quantitative reverse transcription-polymerase chain reaction. ${ }^{* *} \mathrm{P}<0.01$, vs normal tissue; ${ }^{\#} \mathrm{P}<0.05$, vs HK-2 cells. miR, microRNA; RCC, renal cell carcinoma.

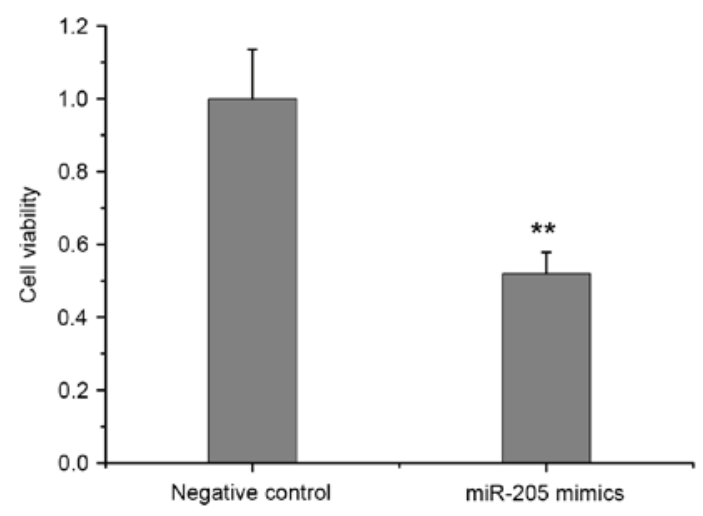

Figure 2. Viability of ACHN cells transfected with miR-205 mimics and the negative control which was determined by methyl thiazolyl tetrazolium (MTT) assay. ${ }^{* *} \mathrm{P}<0.01$, vs negative control. miR, microRNA.

values of proteins. GAPDH was used as a reference gene. Western blotting analysis was repeated three times.

Statistics analysis. Differences were analyzed using Student's t-test. All experiments were repeated at least three times and data were expressed as mean \pm standard deviation. Statistical analysis was performed by t-test using SPSS 17.0 software (SPSS, Inc., Chicago, IL, USA). $\mathrm{P}<0.05$ was considered to indicate a statistically significant difference.

\section{Results}

miR-205 level in RCC tissues and cells. miR-205 expression levels in RCC tissue, normal tissue adjacent to carcinoma, RCC cells, and HK-2 normal renal cells were evaluated using RT-PCR and results are presented in Fig. 1. It was determined that the miR-205 expression level in normal tissue adjacent to carcinoma was $\sim 2$-fold higher than that in RCC tissue $(\mathrm{P}=0.0036)$. The miR-205 expression level in HK-2 normal renal cells was significantly higher compared with $\mathrm{RCC}$ cells, including ACHN ( $\mathrm{P}=0.01)$, 769-p $(\mathrm{P}=0.011)$ and Caki-1 $(\mathrm{P}=0.012)$. The ACHN cells expressed the lowest levels of miR-205 among the cell lines. 


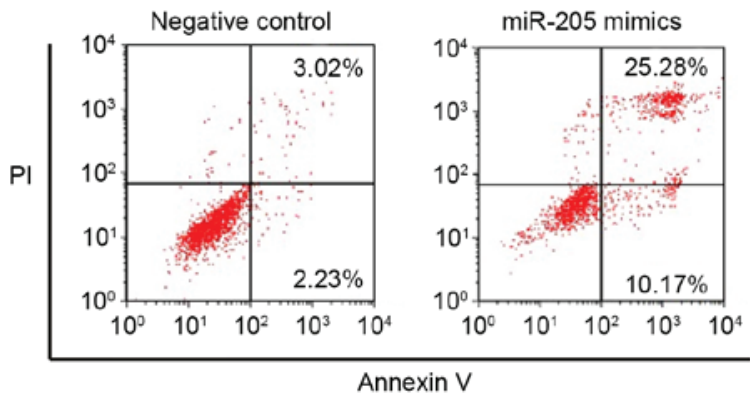

Figure 3. Apoptosis of ACHN cells transfected with miR-205 mimics and the negative control which was assessed by Annexin V-fluorescein isothiocyanate/PI staining. PI, propidium iodide; miR, microRNA.

$m i R$-205 reduces $R C C$ cell viability. Fig. 2 shows the viability of ACHN cells transfected with miR-205 mimics and the negative control, which was determined by MTT assay. When compared with the negative control, the elevation of intracellular miR-205 expression induced by the miR-205 mimics significantly reduced the proliferation of ACHN cells $(\mathrm{P}=0.0059$; Fig. 2).

miR-205 increases the apoptotic rate of RCC cells. Annexin V-FITC/PI staining was used to assess the apoptotic rate of negative control RCC cells and those transfected with miR-205 mimics. Fig. 3 presents the apoptosis of ACHN cells transfected with miR-205 mimics and the negative control. Following the transfection of miR-205 mimics, the number of apoptotic ACHN cells was increased compared with the negative control group, suggesting that the upregulation of miR-205 expression induced the apoptosis of RCC cells.

miR-205 reduces the migration and invasion ability of $R C C$ cells. The migration and invasion ability of ACHN cells transfected with miR-205 mimics and the negative control were evaluated by Transwell assay (Fig. 4). For cell migration, the number of cells migrating to the lower chamber was significantly reduced in cells transfected with miR-205 mimics $(86.83 \pm 12.39)$ compared with the negative control (209.75 $\pm 36.48 ; \mathrm{P}=0.0032)$. In the cell invasion assay, the number of invading cells was significantly reduced in cells transfected with miR-205 mimics $(46.79 \pm 5.28)$ compared with the negative control cells (103.56 $\pm 15.43 ; \mathrm{P}=0.0029)$. These results indicated that the increased level of intracellular miR-205 may inhibit the migration and invasion ability of RCC cells.

Effect of miR-205 on the expression levels of E2F1, Bcl-2, E-cadherin, vimentin and PTEN/AKT in RCC cells. The expression of these signaling molecules in ACHN cells was affected by the transfection of miR-205 mimics and was assessed by western blot analysis (Fig. 5). GADPH was used as an internal control and similar blots of GADPH were observed in all groups, validating the reliable determination protein expression levels. Notably, fainter blots of E2F1 and Bcl-2 were observed in ACHN cells transfected with miR-205 mimics comparing to their darker blots in cells transfected with the negative control (Fig. 5A). This qualitative result was verified by the quantification of the protein levels, indicating that transfection with miR-205 mimics may significantly reduce the expression levels of E2F1 ( $\mathrm{P}=0.0042)$

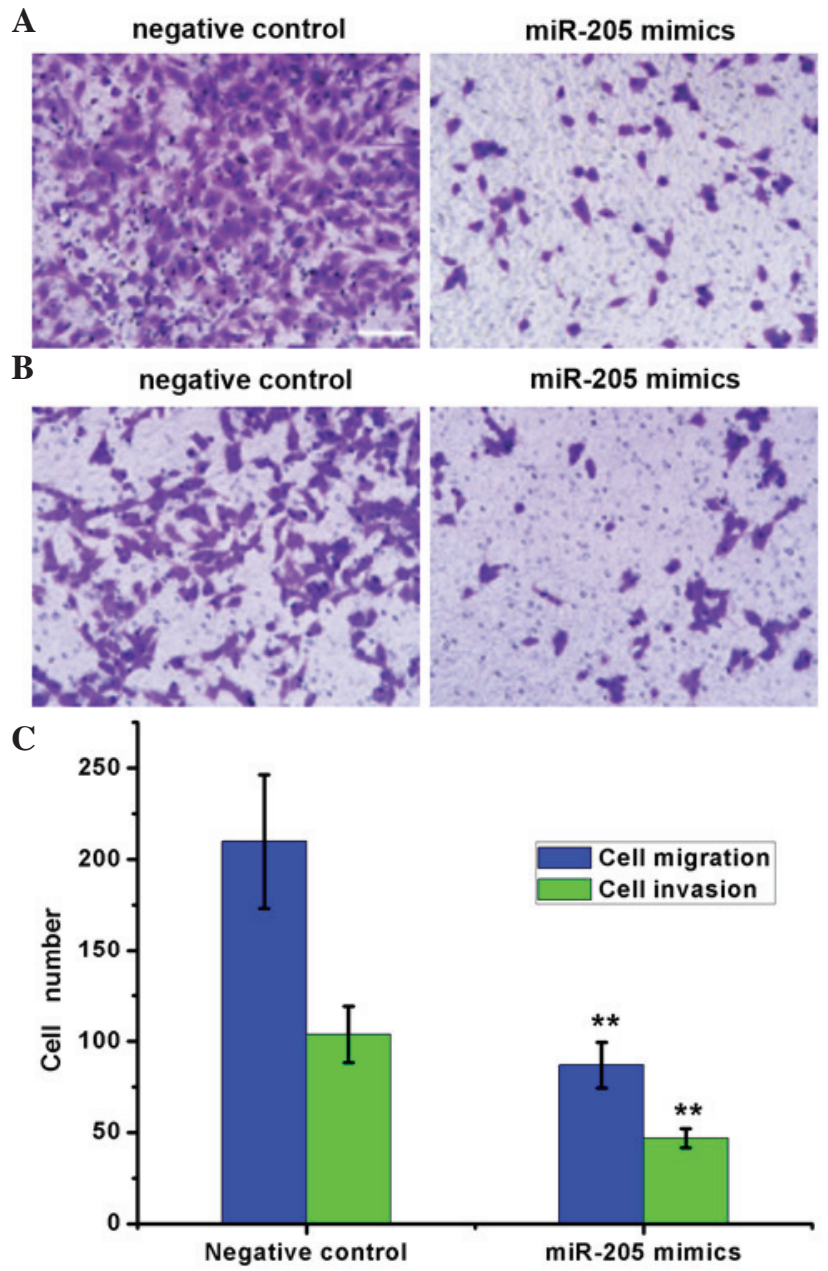

Figure 4. Images of ACHN cells transfected with miR-205 mimics and negative control in the lower chamber of the Transwell assay for the evaluation of (A) cell migration and (B) invasion. Magnification, x200. (C) Quantification of cell migration and invasion. ${ }^{* *} \mathrm{P}<0.01$ vs. negative control. Cells were stained with crystal violet. miR, microRNA.

and $\mathrm{Bcl}-2(\mathrm{P}=0.0047)$ in RCC cells compared with the negative control (Fig. 5A).

ACHN cells transfected with miR-205 mimics exhibited higher expression levels of E-cadherin $(\mathrm{P}=0.0019)$ and reduced expression levels of vimentin $(\mathrm{P}=0.0022)$ compared with the negative control (Fig. 5B). Therefore, miR-205 upregulated the expression of E-cadherin and downregulated the expression of vimentin in RCC cells.

No significant difference between the AKT expression level in ACHN cells transfected with miR-205 mimics and the negative control was identified (data not shown). However, the expression level of PTEN was significantly greater $(\mathrm{P}=0.0085$; Fig. 5C), and that of p-AKT was significantly reduced ( $\mathrm{P}=0.001$; Fig. 5C), in cells transfected with miR-205 mimics compared with the negative control. Therefore, the transfection of miR-205 mimics into RCC cells increased the protein expression of PTEN and reduced the phosphorylation of AKT.

\section{Discussion}

The abnormal expression of miR-205, which may be associated with various types of tumor, is involved in tumorigenesis and 
tumor progression. The present study demonstrated that the expression of miR-205 in RCC tissue was reduced compared with normal tissue adjacent. Previous studies have revealed that miR-205 expression levels were reduced in ACHN cells compared with HK-2 normal renal cells $(10,11)$. miR-205 has also been previously demonstrated to suppress the expression of SRC proto-oncogene, LYN proto-oncogene and YES proto-oncogene 1, which subsequently leads to the inhibition of tumor development (11). The current determined the expression levels of miR-205 using RT-PCR. As ACHN cells exhibited the lowest miR-205 expression level they were used as the model RCC cells in subsequent experiments.

The transfection of miR-205 mimics increased the expression level of miR-205 in RCC cells. It was determined that miR-205 inhibited the proliferation of RCC cells by inducing their apoptosis, and reducing their migration and invasion ability. The expression levels of certain signaling molecules that may be involved in these processes were determined using western blot analysis.

The anabolism of proteins in tumor cells is greater than their catabolism, leading to continuous cell division in tumors. The infinite proliferation of the tumor leads to gradual disease progression. Therefore, reducing cell proliferation and inducing apoptosis of tumor cells may be an effective way to inhibit tumor development. As nuclear transcription factors, the E2F protein family is important for the transcriptional control of cell cycle. E2F1 facilitates the progression of cells from $\mathrm{G} 1$ to $\mathrm{S}$ phase and, additionally, blocks cell apoptosis. A previous study reported that the E2F1 protein expression level is high in suprarenal epithelioma (12). Cell apoptosis is also strictly regulated by various genes. The Bcl-2 family has been extensively investigated. Bcl-2 was initially identified in patients with leucocythemia as a factor able to promote the growth and proliferation of tumor cells. Additionally, it has been established that it may be overexpressed in RCC tissue, and may contribute to the genesis and development of RCC (13). Downregulation of the expression levels of E2F1 and Bcl-2 by miR-205 may, therefore, facilitate the apoptosis of RCC cells. A previous investigation using a luciferase reporter gene in melanoma cells determined that E2F1 is a target gene of miR-205 (14). An increase in the miR-205 expression level in the previous study, directly inhibited the expression of E2F1 and lead to reduced AKT phosphorylation, a key factor in the phosphatidylinositol 3-kinase (PI3K)/AKT signaling pathway (14). Therefore, the proliferation and clone formation of melanoma cells was decreased and the apoptosis of melanoma cells was increased by miR-205 (14). In addition, miR-205 was previously demonstrated to inhibit the proliferation and cell cycle progression of triple negative breast cancer (15). The present study confirmed that elevation of intracellular miR-205 level inhibited the proliferation of RCC cells and promoted their apoptosis, potentially through downregulating the expression levels of E2F1 and Bcl-2.

As important biological characteristics of tumor cells, migration and invasion are primary causes of disease recurrence following surgical intervention, chemotherapy and/or radiotherapy. Epithelial-mesenchymal transition (EMT) is a crucial process involved in migration and invasion. During EMT, tumor cells lose their epithelial phenotype and acquire the phenotype of mesenchymal cells, resulting in the loss
A
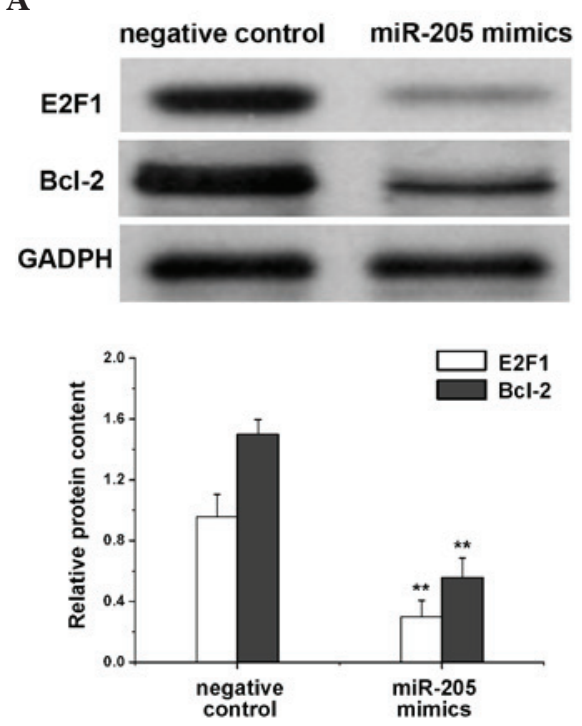

B negative control miR-205 mimics
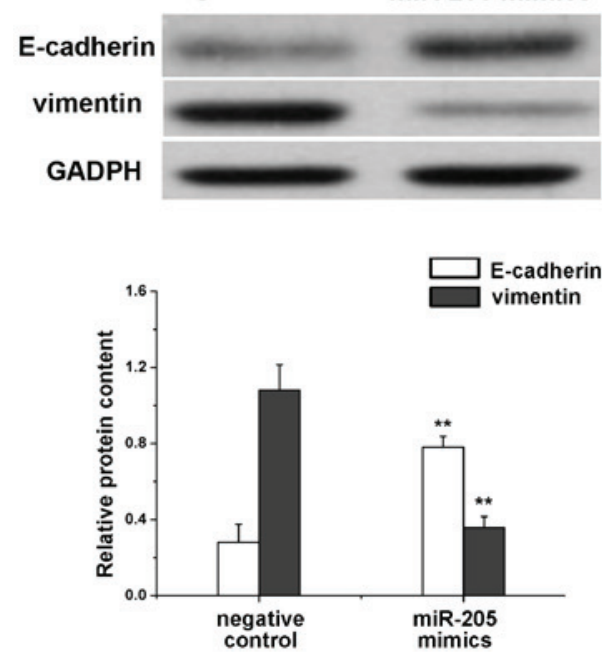

C
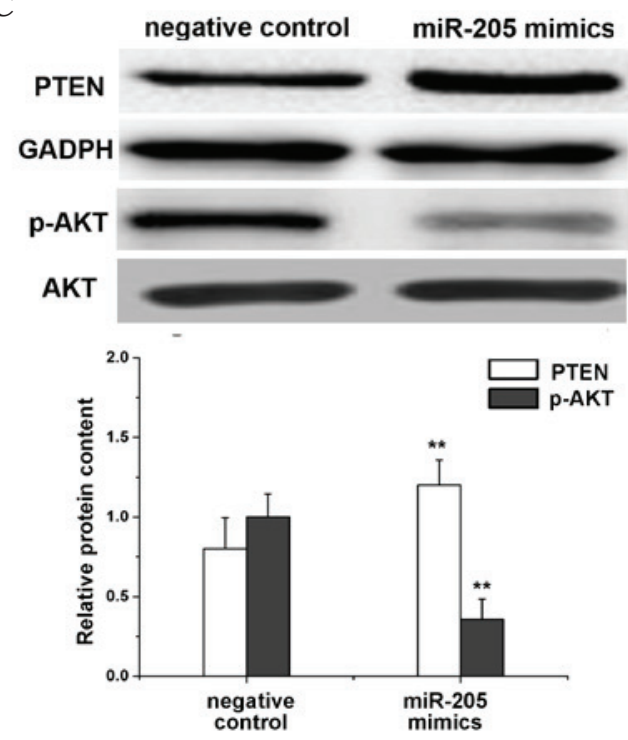

Figure 5. Western blotting and the respective quantification for (A) E2F1/Bcl-2, (B) E-cadherin/vimentin and (C) PTEN/AKT in ACHN cells transfected with miR-205 mimics and the negative control. ${ }^{* *} \mathrm{P}<0.01$ vs. negative control. miR, microRNA; E2F1, E2F transcription factor 1; Bcl-2, B-cell lymphoma-2; PTEN, phosphatase and tensin homolog; p-AKT, phosphorylated AKT serine/threonine kinase 1. 
of the polarity of epithelial cells, reduced contact between surrounding and matrix cells, decreased intercellular interaction and the increased cell migration $(16,17)$. Previous studies have determined that the downregulated expression of E-cadherin, as a cell adhesion molecule, and the upregulated expression of vimentin, a mesenchymal marker, contribute to the molecular characteristics of EMT $(18,19)$. The decreased expression level of E-cadherin and increased level of vimentin reduce intercellular adhesion, and facilitate the invasion capacity of tumor cells from the primary tumor site leading to their subsequent infiltration and diffusion. Therefore, the migration and invasion of tumor cells may be inhibited by reducing the expression levels of EMT-associated proteins. In a previous study using 769-p RCC cells, vimentin was overexpressed and the invasion ability of tumor cells was increased (20). The elevated expression level of miR-205 in melanoma cells and esophageal squamous cell carcinoma cells may upregulate the expression of E-cadherin and downregulate the expression of vimentin, subsequently reducing the migration and invasion of tumor cells $(8,21)$. Therefore, it was hypothesized that miR-205 may inhibit the migration and invasion of RCC cells through a reduction in the expression levels of E-cadherin and vimentin, which was verified in the present study using a Transwell assay and western blotting analysis following transfection of RCC cells with miR-205 mimics.

The PI3K/AKT signaling pathway is important for the genesis and development of various human carcinomas by promoting cell growth, apoptosis, cell cycle progression, angiogenesis, cell migration and invasion. Consequently, the inhibition of the PI3K/AKT signaling pathway may improve cancer therapy. PTEN dephosphorylates phosphatidylinositol $(3,4,5)$-trisphosphate (PIP3) to produce phosphatidylinositol $(4,5)$-bisphosphate (PIP2), thus, negatively regulating the PI3K/AKT signaling pathway. In RCC, the inactivation of PTEN has been observed to increase activation of the P3IK/AKT signaling pathway (22). Zhang et al (23) reported that PTEN is a target gene of miR-205, and the transfection of miR-205 mimics into endometrial cancer cells enhanced the expression of PTEN and phosphorylation of AKT, and inhibited the apoptosis of tumor cells (23). However, in non-small cell lung carcinoma and nasopharyngeal carcinoma cells, the downregulated expression of miR-205 was identified to significantly increase the expression level of PTEN and promote the phosphorylation of AKT $(24,25)$. The effect of miR-205 on the expression of PTEN, phosphorylation of AKT and the associated biological behavior is established, however, the positive or negative regulation exerted by miR-205 on PTEN and p-AKT is contradictory between distinct types of tumor cells. The present study determined, through the use of a Transwell assay and western blotting, that miR-205 promoted the apoptosis of RCC cells and suppressed their proliferation, migration and invasion. miR-250 also increased the PTEN expression levels and inhibited the phosphorylation of AKT.

In conclusion, miR-205 is important for the genesis and development of RCC. The present study demonstrated that miR-205 was expressed in low levels in RCC tissues and cell lines; and this may contribute to the aggressive biological behavior of RCC. Following the transfection of miR-205 mimics into RCC cells, miR-205 promoted the apoptosis of RCC cells and suppressed their proliferation, migration and invasion, which may be mediated by effects on the PTEN/AKT signaling pathway. E2F1, Bcl-2 and vimentin were negatively regulated by miR-205 and E-cadherin was positively regulated. The present study may provide future guidelines for the development of miRNA-associated therapeutic agents for the treatment of RCC.

\section{References}

1. Abdellah A, Selma K, Elamin M, Asmae T, Lamia R, Abderrahmane M, Sanaa el M, Hanan E, Tayeb K and Noureddine B: Renal cell carcinoma in children: Case report and literature review. Pan Afr Med J 20: 84, 2015.

2. Kostrzewa M, Zyła M, Władziński J, Stetkiewicz T, Stachowiak G and Wilczyński JR: Metastases of renal clear cell carcinoma to ovary-case report and review of the literature. Eur J Gynaecol Oncol 36: 219-222, 2015.

3. Ye DW and Zhang HL: Critical appraisal of sorafenib in the treatment of Chinese patients with renal cell carcinoma. Onco Targets Ther 7: 925-935, 2014

4. Gottardo F, Liu CG, Ferracin M, Calin GA, Fassan M, Bassi P, Sevignani C, Byrne D, Negrini M, Pagano F, et al: Micro-RNA profiling in kidney and bladder cancers. Urol Oncol 25: 387-392, 2007.

5. Chow TF, Youssef YM, Lianidou E, Romaschin AD, Honey RJ, Stewart R, Pace KT and Yousef GM: Differential expression profiling of microRNAs and their potential involvement in renal cell carcinoma pathogenesis. Clin Biochem 43: 150-158, 2010.

6. Yi Z, Fu Y, Zhao S, Zhang X and Ma C: Differential expression of miRNA patterns in renal cell carcinoma and nontumorous tissues. J Cancer Res Clin Oncol 136: 855-862, 2010.

7. Lim LP, Glasner ME, Yekta S, Burge CB and Bartel DP: Vertebrate microRNA genes. Science 299: 1540, 2003.

8. Matsushima K, Isomoto H, Yamaguchi N, Inoue N, Machida H, Nakayama T, Hayashi T, Kunizaki M, Hidaka S, Nagayasu T, et al: MiRNA-205 modulates cellular invasion and migration via regulating zinc finger E-box binding homeobox 2 expression in esophageal squamous cell carcinoma cells. J Transl Med 9: 30, 2011.

9. Gandellini P, Folini M, Longoni N, Pennati M, Binda M, Colecchia M, Salvioni R, Supino R, Moretti R, Limonta P, et al: miR-205 Exerts tumor-suppressive functions in human prostate through down-regulation of protein kinase Cepsilon. Cancer Res 69: 2287-2295, 2009.

10. Chen Z, Tang ZY, He Y, Liu LF, Li DJ and Chen X: miRNA-205 is a candidate tumor suppressor that targets ZEB2 in renal cell carcinoma. Oncol Res Treat 37: 658-664, 2014.

11. Majid S, Saini S, Dar AA, Hirata H, Shahryari V, Tanaka Y, Yamamura S, Ueno K, Zaman MS, Singh K, et al: MicroRNA-205 inhibits Src-mediated oncogenic pathways in renal cancer. Cancer Res 71: 2611-2621, 2011.

12. Gao Y, Fan Y, Chen W, Huang Q, Ai Q, Ni D, Ma X and Zhang X: Effect of E2Fl knockdown on proliferation and invasion of clear cell renal cell carcinoma cell line Caki-2. Zhong Hua Shi Yan Wai Ke Za Zhi She: 333-335, 2013 (In Chinese).

13. Li Y, Zhang J, Ge D, Yang Y and Yu J: Expression and significance of E-cadherin and Bcl-2 in early renal carcinoma and adjacent tissues of different levels of distance. J Contemp Urol Reprod Onco: 108-111, 2011 (In Chinese).

14. Dar AA, Majid S, de Semir D, Nosrati M, Bezrookove V and Kashani-Sabet M: miRNA-205 suppresses melanoma cell proliferation and induces senescence via regulation of E2F1 protein. J Biol Chem 286: 16606-16614, 2011.

15. Piovan C, Palmieri D, Di Leva G, Braccioli L, Casalini P, Nuovo G, Tortoreto M, Sasso M, Plantamura I, Triulzi T, et al: Oncosuppressive role of p53-induced miR-205 in triple negative breast cancer. Mol Oncol 6: 458-472, 2012.

16. Yang JD, Nakamura I and Roberts LR: The tumor microenvironment in hepatocellular carcinoma: Current status and therapeutic targets. Semin Cancer Biol 21: 35-43, 2011.

17. Ogunwobi OO and Liu C: Therapeutic and prognostic importance of epithelial-mesenchymal transition in liver cancers: Insights from experimental models. Crit Rev Oncol Hematol 83: 319-328, 2012.

18. Yamada S, Okumura N, Wei L, Fuchs BC, Fujii T, Sugimoto H, Nomoto S, Takeda S, Tanabe KK and Kodera Y: Epithelial to mesenchymal transition is associated with shorter disease-free survival in hepatocellular carcinoma. Ann Surg Oncol 21: 3882-3890, 2014. 
19. Hou KZ, Fu ZQ and Gong H: Chemokine ligand 20 enhances progression of hepatocellular carcinoma via epithelial-mesenchymal transition. World J Gastroenterol 21: 475-483, 2015.

20. Fang Y, Wei J, Cao J, Zhao H, Liao B, Qiu S, Wang D, Luo J and Chen W: Protein expression of ZEB2 in renal cell carcinoma and its prognostic significance in patient survival. PLoS One 8: e62558, 2013.

21. Liu S, Tetzlaff MT, Liu A, Liegl-Atzwanger B, Guo J and Xu X: Loss of microRNA-205 expression is associated with melanoma progression. Lab Invest 92: 1084-1096, 2012.

22. Hager M, Haufe H, Lusuardi L, Schmeller N and Kolbitsch C: PTEN, pAKT, and pmTOR expression and subcellular distribution in primary renal cell carcinomas and their metastases. Cancer Invest 29: 427-438, 2011.
23. Zhang G, Hou X, Li Y and Zhao M: MiR-205 inhibits cell apoptosis by targeting phosphatase and tensin homolog deleted on chromosome ten in endometrial cancer Ishikawa cells. BMC Cancer 14: 440, 2014.

24. Cai J, Fang L, Huang Y, Li R, Yuan J, Yang Y, Zhu X, Chen B, Wu J and Li M: miR-205 targets PTEN and PHLPP2 to augment AKT signaling and drive malignant phenotypes in non-small cell lung cancer. Cancer Res 73: 5402-5415, 2013.

25. Qu C, Liang Z, Huang J, Zhao R, Su C, Wang S, Wang X, Zhang R, Lee MH and Yang H: MiR-205 determines the radioresistance of human nasopharyngeal carcinoma by directly targeting PTEN Cell Cycle 11: 785-796, 2012. 